\title{
Single and Multi Container Maintenance Model: A Fuzzy Geometric Programming Approach
}

\author{
Ghanshaym Singha Mahapatra \\ Department of Mathematics \\ Bengal Engineering and Science University, Shibpur \\ Howrah, West Bengal 711103, India \\ Tel: 91-943-313-5327 E-mail: g_S_mahapatra@yahoo.com \\ Tapan Kumar Roy (Corresponding author) \\ Department of Mathematics \\ Bengal Engineering and Science University, Shibpur \\ Howrah, West Bengal 711103, India \\ Tel: 91-943-265-8432 E-mail: roy_t_k@yahoo.co.in
}

This research is financially supported by C.S.I.R., India, through research project (25(0151)/06/EMR-II dated: 11.09.06) in the Department of Mathematics, Bengal Engineering and Science University, Shibpur. This support is great fully acknowledged.

\begin{abstract}
In this paper, single and multi container maintenance model under limited time interval of interest is introduced in fuzzy environment. These fuzzy models are solved by fuzzy geometric programming technique. Here fuzzy geometric programming is discussed through fuzzy decision-making processes with three different operators, max-min, max-average mean, and max-geometric mean. These operators are applied in the single-container maintenance model in fuzzy environment. Fuzzy multi-container maintenance model through max-average mean operator which is solved by modified geometric programming. Numerical examples are shown to illustrate the fuzzy models.
\end{abstract}

Keywords: Maintenance, Fuzzy set, Fuzzy geometric programming, Replacement, Modified geometric programming

\section{Introduction}

The reliability is an important research topic in engineering and operation research. Maintenance is one of the effective ways of increasing the durability of a system. However, the repair will improve the availability of a single equipment system. It is, therefore, necessary to estimate the optimum maintenance that will minimize the system downtime and hence maximize the system durability. To perform a system impeccably it is required to maintain the system up to the desire label and finally, replacement is needed when it fails to do so. It is important to find out the exact critical time to replace the system. To keep a system in operating condition, the different activities of maintenance is necessary. Maintenance action varies between the corrective maintenance $(\mathrm{CM})$ and the preventive maintenance (PM). $C M$ is maintained up to the desired level of maintenance cost but proved to be ineffective, and then the replacement of the system is required. PM cost is low for low level of PM effort but the expected CM cost is high when CM cost decreases and the PM effort increases, the total $(\mathrm{PM}+\mathrm{CM})$ cost decreases initially and then increases with increasing PM effort. This implies that there is an optimal level of PM effort to minimize the total maintenance cost. Minimization of cost per unit time is one approach to determine the optimal maintenance policy.

Geometric Programming (GP) is one of the effective methods to solve a particular type of non-linear programming problem. Geometric Programming is introduced by Zener (1971), and it is further developed by Duffin, Peterson and Zener (1967). In GP method, Degree of Difficulty (DD) plays a significant role. It is defined as DD = number of terms in objective and constraint functions - number of primal decision variables - 1 . If $\mathrm{DD}=0$, then dual variables can be determined uniquely from the normality and orthogonality conditions. In 1987, Cao (1987) first introduced FGP. Cao (1993) 
discussed fuzzy geometric programming with zero degree of difficulty. Later on a brief discussion on FGP is found in the book by Cao (2002). In case of higher values of DD, it is not very easy to solve the problem by GP technique. If DD > 0 , there are infinite number of solutions of the system of constraint equations in the dual problem. Abou-et-ata and Kotb (1997) and Islam and Roy (2005) introduced a new idea on GP which is called modified geometric programming (MGP) by reducing the DD to avoid the numerical difficulty. GP method is used rarely to solve the maintenance problem. Govil (1992) discussed optimal maintainability problem using the geometric programming technique. But fuzzy replacement model through FGP is very rare in literature.

Let the item is a container and a failure occurs when there is a leak in the container. As the item ages, the failure rate increases due to degradation of material of the container made. In this paper, single and multi container maintenance models are taken in fuzzy environment. Here the failure distribution of the containers is Weibull distribution with two parameters. Fuzzy single container maintenance model solved by fuzzy geometric programming technique through fuzzy decision-making process namely max-min, max-arithmetic mean and max-geometric mean operator. For higher degree of difficulty of multi-container maintenance model, fuzzy geometric programming technique through max-arithmetic mean operator is solved by MGP. Finally, numerical examples are given to show the above approaches of FGP on container maintenance models to obtain an optimal maintenance policy of the system in fuzzy environment.

\section{Model formulation}

$\begin{array}{ll}\text { Notations } & \\ T & \text { expected cycle length } \\ J c(T) & \text { total expected maintenance cost per unit time } \\ M(T) & \text { time required for replacing failed/nonfailed item } \\ P_{J c} & \text { the tolerance of Jc(T) } \\ C_{p} & \text { cost of a preventive maintenance } \\ C_{r} & \text { cost of corrective maintenance } \\ L & \text { time interval of interest } \\ P_{L} & \text { the tolerance of L } \\ m & \text { fixed factor }(<1) \\ \text { Parameters for ith }(i=1,2, \cdots) \text { containers } \\ T_{i} & \text { expected cycle length } \\ J_{c}\left(T_{i}\right) & \text { total expected maintenance cost per unit time } \\ M\left(T_{i}\right) & \text { time required for replacing failed/nonfailed item } \\ P_{J c i} & \text { the tolerance of Jc(T) } \\ C_{p i} & \text { cost of a preventive maintenance } \\ C_{r i} & \text { cost of corrective maintenance } \\ L^{\prime} & \text { time interval of interest } \\ P_{L^{\prime}} & \text { the tolerance of L } \\ m_{i} & \text { fixed factor }(<1) \\ \end{array}$

\subsection{Crisp model of single container maintenance problem}

Let the item is a container and a failure occurs whenever there is a leakage in the container. The failure distribution $\mathrm{F}(\mathrm{t})$ is taken as Weibull distribution with shape parameter $\alpha$ and scale parameter $\beta$. Then the expected cost (see Appendix-I) of maintenance under the time interval $\mathrm{L}$ is

$$
\begin{array}{lcc}
\operatorname{Min} J c(T) & = & \frac{C_{p}}{T}+\left(\frac{C_{r}}{\beta^{\alpha}}\right) T^{\alpha-1} \\
\text { subject to } M(T) & = & m T \leq L \\
& & T>0
\end{array}
$$

It should be considered that $M(T)=m T$, the time required for a corrective maintenance action (replacing failed item by new one) and preventive maintenance action (replacing nonfailed item by new one). Where $\alpha>1$ (if $\alpha \leq 1$ it is decreasing or constant failure rate).

\subsection{Crisp model of multi-containers maintenance problem}

Let there are multi containers of same material but different in shape and size. The failure distribution $F(t)$ is taken as Weibull distribution with shape parameter $\alpha$ and scale parameter $\beta$. The expected cost of maintenance of each container under the total time of interval is formulated as multi-item multi-objective problem as follows

$$
\operatorname{Min} J c_{i}\left(T_{i}\right)=\frac{C_{p i}}{T}+\left(\frac{C_{r i}}{\beta^{\alpha}}\right) T_{i}^{\alpha-1} \quad \text { for } i=1,2,3, \cdots, n
$$




$$
\text { subject to } M\left(T_{1}, T_{2}, \cdots, T_{n}\right)=\sum_{i=1}^{n} m_{i} T i \leq L^{\prime}
$$

\subsection{Fuzzy model of single container maintenance problem}

Defining the container model (2.1), including the formulation of an objective and constraint functions is very complicated decision making process. Determining objective goal, the cost of maintenance as well as constraint goal time of interval of interest may be uncertain but non-stochastic in nature. Therefore the maintenance of container problem (2.1) can be represented by fuzzy non-linear programming to make the model more flexible and acceptable to the human decision process. Therefore in fuzzy environment the container problem (2.1) becomes

$$
\begin{array}{lcc}
\tilde{M} \text { in } J C(T) & = & \frac{C_{p}}{T}+\left(\frac{C_{r}}{\beta^{\alpha}}\right) T^{\alpha-1} \leq J C \\
\text { subject to } M(T) & = & m T \leq L \\
& & T>0
\end{array}
$$

Here objective and constraint functions are posynomial form. So we can use geometric programming to solve this problem.

\subsection{Fuzzy model of multi-containers maintenance problem}

The maintenance of container (2.2) can also be represented by fuzzy non-linear programming. Therefore in fuzzy environment multi-item containers problem (2.2) becomes

$$
\begin{aligned}
& \tilde{\operatorname{Min} J c_{1}}\left(T_{1}\right)=\frac{C_{p 1}}{T_{1}}+\left(\frac{C_{r 1}}{\beta^{\alpha}}\right) T_{1}^{\alpha-1} \underset{\sim}{\leq} J c_{1}
\end{aligned}
$$

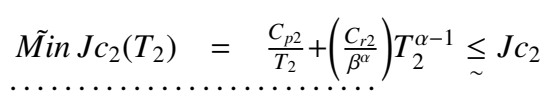

$$
\begin{aligned}
& \tilde{\operatorname{Min} J c_{n}}\left(T_{n}\right)=\frac{C_{p n}}{T_{n}}+\left(\frac{C_{r n}}{\beta^{\alpha}}\right) T_{n}^{\alpha-1} \leq J c_{n} \\
& \text { subject to } M\left(T_{1}, T_{2}, \cdots, T_{n}\right)=\sum_{i=1}^{n} m_{i} T i \underset{\sim}{\leq} L^{\prime} \\
& T_{i}>0 \quad \text { for } i=1,2,3, \cdots, n
\end{aligned}
$$

\section{Prerequisties mathematics}

Fuzzy sets is introduced by Zadeh (1965) as a mathematical way of representing impreciseness or vagueness in everyday life.

\subsection{Fuzzy Set}

A fuzzy set $\tilde{A}$ of the universe of discourse $X$ is defined as the following set of pairs $\tilde{A}=\left\{\left(x, \mu_{\tilde{A}}(x)\right): x \in X\right\}$. Here $\mu_{\tilde{A}}: X \rightarrow[0,1]$ is a mapping called the membership function of the fuzzy set $\tilde{A}$ and $\mu_{\tilde{A}}(x)$ is called the membership value or degree of membership of $x \in X$ in the fuzzy set $\tilde{A}$. The larger $\tilde{A}$ and $\mu_{\tilde{A}}(x)$ is the stronger the grade of membership in $\tilde{A}$.

\subsection{Normal Fuzzy Set}

A fuzzy set $\tilde{A}$ of the universe of discourse $X$ is called a normal fuzzy set implying that there exists at least one $x \in X$ such that $\mu_{\tilde{A}}(x)=1$

\subsection{Fuzzy Decision Making}

In this real world most of the decision making problems take place in a fuzzy environment. Here objective goal, constraints and the consequences of possible actions are not known precisely. Under this observation, Bellman, et. al. (1970) has introduced three basic concepts. They are fuzzy objective goal, fuzzy constraint and fuzzy decision based on fuzzy goal and constraint. The conceptual framework for decision making has been introduced in a fuzzy environment.

Let $\mathrm{X}$ is a given set of possible alternatives which contains the solution of a decision making problem in fuzzy environment. Fuzzy decision making problem may be taken as

$\tilde{G}_{0}$ : Optimize fuzzy objective function

Subject to

$\tilde{C}_{j}$ : Fuzzy constraints for $j=1,2, \cdots, m$

\subsubsection{Fuzzy goal}

Here fuzzy goal $\tilde{G}_{0}=\left\{\left(x, \mu_{\tilde{G}_{0}}(x)\right): \forall x \in X\right\}$ is a fuzzy set on possible alternatives $X$ characterized by it membership function $\mu_{\tilde{G}_{0}}: X \rightarrow[0,1]$. 


\subsubsection{Fuzzy constraints}

Here fuzzy Constraints $\tilde{C}_{j}=\left\{\left(x, \mu_{\tilde{C}_{j}}(x)\right): \forall x \in X\right\}$ is a fuzzy set on possible alternatives $X$ characterized by it membership function $\mu_{\tilde{C}_{j}}: X \rightarrow[0,1]$. for $j=1,2, \cdots, m$

\subsubsection{Fuzzy decision}

For fuzzy decision making problem, fuzzy goal $\tilde{G}_{0}$ and m number of fuzzy constraints $\tilde{C}_{j}(j=1,2, \cdots, m)$ are expected to be satisfied simultaneously. According to Bellman and Zadeh (1970) fuzzy decision through fuzzy objective goal and fuzzy constraints can be found as follows

Fuzzy decision based on max-min operator $\tilde{D}_{m}=\left\{\left(x, \mu_{\tilde{D}_{m}}(x)\right): \forall x \in X\right\}$ is a fuzzy set defined as $\tilde{D}_{m}=\tilde{G}_{0} \underset{j=1}{\bigcap_{j}} \tilde{C}_{j}$

It is characterized by $\mu_{\tilde{D}_{m}}(x)=\min _{\forall j=1,2, \cdots, m}\left(\mu_{\tilde{G}_{0}}(x), \mu_{\tilde{C}_{j}}(x)\right) \forall x \in X$

So, optimal decision vector $x^{*}$ has been derived from

$$
\operatorname{Max}_{\tilde{D}_{m}}\left(x^{*}\right)=\max _{x \in X}\left(\min _{\forall j=1,2, \cdots, m}\left(\mu_{\tilde{G}_{0}}(x), \mu_{\tilde{C}_{j}}(x)\right)\right)
$$

There are two other aggregation patterns, which are based on arithmetic mean and geometric mean operators.

Fuzzy decision based on arithmetic mean operator is a fuzzy set $\tilde{D}_{a}=\left\{\left(x, \mu_{\tilde{D}_{a}}(x)\right): \forall x \in X\right\}$.

Here $\mu_{\tilde{D}_{a}}(x)=\left(\left(\mu_{G_{0}}(x)+\sum_{j=1}^{m} \mu_{C_{j}}(x)\right) /(m+1)\right) \forall x \in X$

So, optimal decision vector $x^{*}$ has been derived from $\operatorname{Max} \mu_{\tilde{D_{a}}}\left(x^{*}\right)=\left(\max _{x \in X}\left(\mu_{\widetilde{G_{0}}}(x)+\sum_{j=1}^{m} \mu_{{\widetilde{C_{j}}}_{j}}(x)\right) /(m+1)\right)$

Fuzzy decision based on geometric mean operator is a fuzzy set $\tilde{D_{P}}=\left\{\left(x, \mu_{\tilde{D_{P}}}(x)\right): \forall x \in X\right\}$

Here $\mu_{\tilde{D_{P}}}(x)=\left(\mu_{\tilde{G_{0}}}(x) \prod_{j=1}^{m} \mu_{\tilde{C}_{j}}(x)\right)^{\frac{1}{(m+1)}} \forall x \in X$

So, optimal decision vector $x^{*}$ has been derived from $\operatorname{Max}_{\tilde{D}_{a}}\left(x^{*}\right)=\max _{x \in X}\left(\mu_{\tilde{\sigma_{0}}}(x) \prod_{j=1}^{m} \mu_{\tilde{C}_{j}}(x)\right)^{\frac{1}{(m+1)}}$

\section{Geometric Programming}

Geometric Programming(GP) had its beginning in 1961 by Zener. Later on Duffin, Peterson and Zener wrote an authoritative book on GP. The extension of the GP method for negative terms and arbitrary inequalities was achieved by Wilde and Passy, which is known as signomial GP.

The standard signomial GP problem may be taken as:

Find $t=\left(t_{1}, t_{2}, \cdots, t_{n}\right)^{T}$ of the following problem:

Minimize $g_{0}(t)=\sum_{k=1}^{T_{0}} \sigma_{0 k} c_{0 k} \prod_{r=1}^{n} t_{r}^{\alpha_{0 k r}}$

Subject to $g_{j}(t)=\sum_{k=1}^{T_{j}} \sigma_{j k} c_{j k} \prod_{r=1}^{n} t_{r}^{\alpha_{j k r}} \leq \zeta_{j} g_{j}^{0}$ for $j=1,2,3, \cdots, m$

$$
t_{r}>0 \quad \text { for } r=1,2,3, \cdots, n
$$

where $c_{r k}, g_{j}^{0}(>0), \alpha_{r k j}$, sign $\sigma_{j k}= \pm 1, \zeta_{j}= \pm 1(k=1,2,3, \cdots, T j ; j=0,1,2,3, \cdots, n ; r=1,2, \cdots, n)$ are real numbers.

It may be written as a constrained GP problem with Degree of Difficulty

$(D D)=\sum_{j=0}^{m} T_{j}-(n+1)$

The pseudo dual of the problem (4.1) is 
$\operatorname{Maximize} d(w, \lambda) \equiv \zeta_{0}\left[\prod_{j=1}^{m}\left(\frac{c_{0 k}}{w_{0 k}}\right)^{\sigma_{0 k} w_{0 k}} \prod_{k=1}^{m}\left(\frac{c_{j k}}{w_{j k}}\right)^{\sigma_{j k} w_{j k}} \prod_{j=1}^{m} \lambda_{j}^{\zeta_{j} \lambda_{j}}\right]^{\zeta_{0}}$

Subject to

$$
\begin{array}{ll}
\sum_{k=1}^{T_{j}} \sigma_{j k} w_{j k}=\zeta_{j k} \lambda_{j k} & \text { for } j=0,1,2, \cdots, m \\
\sum_{k=1}^{p} \sigma_{j k} \alpha_{j k} w_{j k}=0 & \text { for } r=1,2,3, \cdots, n ; j=1,2,3, \cdots, m \\
w_{j k} \geq 0 & \text { for } j=1,2,3, \cdots, m ; k=1,2,3, \cdots, p \\
\lambda_{0}=1 \text { and } \lambda_{k} \geq 0, k=1,2, \cdots, p &
\end{array}
$$

(Note that it is assumed that the sign of the objective, $\zeta_{0}$ is known)

In real life problem, it is possible to soften the rigid requirements of the decision maker (DM) to minimize the objective function strictly and satisfy the constraints strictly. In this situation, above GP may be taken as following single objective fuzzy geometric programming (SOFGP) problem.

\subsection{Single Objective Fuzzy Geometric Programming with its Solution Procedures}

Min $g_{0}(t)=\sum_{k=1}^{T_{0}} \sigma_{0 k} c_{0 k} \prod_{r=1}^{n} t_{r}^{\alpha_{0 k r}} \underset{\sim}{\leq} g_{0}$

subject to

$$
\begin{array}{ll}
g_{j}(t)=\sum_{k=1}^{T_{j}} \sigma_{j k} c_{j k} \prod_{r=1}^{n} t_{r}^{\alpha_{j k r}} \leq \zeta_{j} g_{j}^{0} & \text { for } j=1,2, \cdots, m \\
t_{r}>0 & \text { for } r=1,2, \cdots, n
\end{array}
$$

Hence the symbol ' $\tilde{M i n}$ ' denotes a relaxed or fuzzy version of 'Min'. It implies that the objective function should be minimized as well as possible near $g_{0}$. Similarly, the symbol ' $\leq$ ' denotes a fuzzy version of ' $\leq$ '. It also implies that the constraints should be well satisfied. Here objective and constraint goals $g_{j}$ with maximum tolerance is $\left(g_{j}^{\prime}-g_{j}^{0}\right)$ for $j=0,1,2, \cdots, m$. These fuzzy requirements may be quantified by eliciting membership functions $\mu_{j}\left(g_{j}(t)\right)$ from the DM for all functions $g_{j}(t)(j=0,1,2, \cdots, m)$. By taking account of the rate of increased membership satisfaction, the DM must determine the subjective membership function $\mu_{j}\left(g_{j}(t)\right)$. It is in general a strictly monotone decreasing linear or non-linear function $u_{j}\left(g_{j}(t)\right)$ with respect to $g_{j}(t)(j=0,1,2, \cdots, m)$. Here for simplicity, linear membership functions are taken.

They are

$$
\mu_{j}\left(g_{j}(t)\right)=\left\{\begin{array}{cl}
1 & \text { if } g_{j}(t) \leq g_{j}^{0} \\
u_{j}\left(g_{j}(t)\right)=\frac{g_{j}^{\prime}-g_{j(t)}}{g_{j}^{\prime}-g_{j}^{0}} & \text { if } g_{j}^{0} \leq g_{j}(t) \leq g_{j}^{\prime} \\
0 & \text { if } g_{j}(t) \geq g_{j}^{\prime}
\end{array} \quad \text { for } j=0,1,2, \cdots, m\right.
$$

Its rough sketch is

$<$ Figure $1>$

As shown in the above figure 1 ,

$g_{j}^{0} \equiv$ the value of $g_{j}(t)$ is such that the grade of membership function $\mu_{j}\left(g_{j}(t)\right)$ is 1 .

$g_{j}^{\prime} \equiv$ the value of $g_{j}(t)$ is such that the grade of membership function $\mu_{j}\left(g_{j}(t)\right)$ is 0 .

$\bar{g}_{j} \equiv$ the intermediate value of $g_{j}(t)$ between $g_{j}^{0}$ and $g_{j}^{\prime}\left(\right.$ i.e. $\left.\bar{g}_{j} \in\left(g_{j}^{0}, g_{j}^{\prime}\right)\right)$ is such that the grade of membership function say $\alpha(\in(0,1))$.

Fuzzy decision making process (Bellman, et.al., 1997) based on fuzzy objective and constraint goals using above said membership functions $\mu_{j}\left(g_{j}(t)\right)(j=0,1,2, \ldots, m)$ the problem of finding the optimal decision vector $t^{*}$ based on three operators (namely Max-min, Max-arithmetic mean, and Max-geometric mean) are as follows:

\subsubsection{Equivalent Crisp GP of SOFGP based on Max-Min Operator:}

Find $t^{*}=\left(t_{1}^{*}, t_{2}^{*}, \cdots, t_{n}^{*}\right)^{T}$ where $\mu_{m}\left(t^{*}\right)=\max _{\forall t>0}\left(\min _{\forall j=0,1,2, \cdots, m} \mu_{j}\left(g_{j}(t)\right)\right)$ 
Following Sakawa (1993), (4.4) is equivalent to $\operatorname{Max} \mu_{0}\left(g_{0}(t)\right)$ subject to

$\mu_{j}\left(g_{j}(t)\right) \geq \mu_{0}\left(g_{0}(t)\right)$ for $i=1,2, \cdots, m$

Considering membership functions (4.3), the problem (4.2) reduces to

$\operatorname{Max} V_{m}(t)=\frac{g_{0}^{\prime}-\sum_{i=1}^{T_{0}} \sigma_{0 i} c_{0 i} \prod_{r=1}^{n} t_{r}^{\alpha_{0 i r}}}{g_{0}^{\prime}-g_{0}^{0}}$

subject to

$\frac{g_{j}^{\prime}-\sum_{i=1}^{T_{j}} \sigma_{j i} c_{j i} \prod_{r=1}^{n} t_{r}^{\alpha_{j i r}}}{g_{j}^{\prime}-g_{j}^{0}} \geq \frac{g_{0}^{\prime}-\sum_{i=1}^{T_{0}} \sigma_{0 i} c_{0 i} \prod_{r=1}^{n} t_{r}^{\alpha_{0 i r}}}{g_{0}^{\prime}-g_{0}^{0}}$ for $j=1,2,3, \cdots, m$

$t_{r}>0 \quad$ for $r=1,2,3, \cdots, n$

so optimal decision variable vector $t^{*}=\left(t_{1}^{*}, t_{2}^{*}, \cdots, t_{n}^{*}\right)^{T}$ with optimal objective value is

$V_{m}^{*}\left(t^{*}\right)=\frac{g_{0}^{\prime}}{g_{0}^{\prime}-g_{0}^{0}}-V_{m}^{\prime}\left(t^{*}\right)$

where $t^{*}=\left(t_{1}^{*}, t_{2}^{*}, \cdots, t_{n}^{*}\right)^{T}$ is optimal decision variable vector of the following GP problem

$\operatorname{Min} V_{m}^{\prime}\left(t^{*}\right)=\frac{1}{g_{0}^{\prime}-g_{0}^{0}} \sum_{i=1}^{T_{0}} \sigma_{0 i} c_{0 i} \prod_{r=1}^{n} t_{r}^{\alpha_{0 i r}}$

subject to

$\frac{g_{0}^{\prime}-g_{0}^{0}}{g_{0}^{\prime} g_{j}^{\prime}-g_{j}^{\prime} g_{0}^{0}} \sum_{i=1}^{T_{j}} \sigma_{j i} c_{j i} \prod_{r=1}^{n} t_{r}^{\alpha_{j i r}}-\frac{g_{0}^{\prime}-g_{0}^{0}}{g_{0}^{\prime} g_{j}^{\prime}-g_{j}^{\prime} g_{0}^{0}} \sum_{i=1}^{T_{0}} \sigma_{0 i} c_{0 i} \prod_{r=1}^{n} t_{r}^{\alpha_{0 i r}} \leq 1$ for $j=1,2,3, \cdots, m$
$t_{r}>0 \quad$ for $r=1,2,3, \cdots, n$

It is a constrained signomial GP with $D D=m T_{0}+\sum_{j=0}^{m} T_{j}-(n+1)$

4.1.2 Equivalent Crisp GP of SOFGP based on Max-Arithmatic Mean Operator

Find $t^{*}=\left(t_{1}^{*}, t_{2}^{*}, \cdots, t_{n}^{*}\right)^{T}$ where

$\mu_{a}\left(t^{*}\right)=\max _{\forall t>0}\left(\sum_{j=0}^{m} \mu_{j}\left(g_{j}(t)\right) /(m+1)\right)$

Considering membership functions(4.3), the problem (4.2) reduces to

$\operatorname{Max}_{a}(t)=\sum_{j=0}^{m} \frac{g_{j}^{\prime}-\sum_{i=1}^{T_{j}} \sigma_{j i} c_{j i} \prod_{r=1}^{n} t_{r}^{\alpha_{j i r}}}{(m+1)\left(g_{j}^{\prime}-g_{j}^{0}\right)}$

subject to $\quad t_{1}>0 \quad$ for $r=1,2,3, \cdots, n$

So optimal decision variable vector $t^{*}=\left(t_{1}^{*}, t_{2}^{*}, \cdots, t_{n}^{*}\right)^{T}$ with optimal objective value is

$V_{a}^{*}\left(t^{*}\right)=\frac{1}{(m+1)}\left(\sum_{j=0}^{m} \frac{g_{j}{ }^{\prime}}{g_{j}^{\prime}-g_{j}^{0}}-V_{a}^{\prime}\left(t^{*}\right)\right)$ where $t^{*}=\left(t_{1}^{*}, t_{2}^{*}, \cdots, t_{n}^{*}\right)^{T}$ is the optimal decision vector of the unconstrained geometric programming problem

$\operatorname{Min} V_{a}^{\prime}(t)=\sum_{j=0}^{m} \frac{1}{g_{j}^{\prime}-g_{j}^{0}} \sum_{i=1}^{T_{j}} \sigma_{j i} c_{j i} \prod_{r=1}^{n} t_{r}^{\alpha_{j i r}}$

subject to $\quad t_{r}>0 \quad$ for $r=1,2,3, \cdots, n$

It is a unconstrained posynomial GP with $D D=\sum_{j=0}^{m} T_{j}-(n+1)$ 
4.1.3 Equivalent Crisp GP of SOFGP based on Max-Geometric Mean Operator

Find $t^{*}=\left(t_{1}^{*}, t_{2}^{*}, \cdots, t_{n}^{*}\right)^{T}$ where

$\mu_{p}\left(t^{*}\right)=\max _{\forall t>0}\left(\prod_{j=0}^{m} \mu_{j}\left(g_{j}(t)\right)\right)^{\frac{1}{(m+1)}}$

Considering memberhip finctions (4.3), the problem (4.2) reduces to

$\operatorname{Max}\left(V_{p}(t)\right)^{m+1}=\left(\prod_{j=0}^{m}\left(\frac{g_{j}^{\prime}-\sum_{i=1}^{T_{j}} \sigma_{j i} c_{j i} \prod_{r=1}^{n} t_{r}^{\alpha_{j i r}}}{g_{j}^{\prime}-g_{j}^{0}}\right)\right)$

subject to $\quad t_{r}>0 \quad$ for $r=1,2,3, \cdots, n$

It can be formulated as an unconstrained signomial GP problem with

$D D=\prod_{j=0}^{m}\left(T_{j}+1\right)-(n+1)$.

Note: 4.1 Through max-geometric mean operator, FGP becomes a crisp GP with higher DD than that through other two operators namely, max-min and max-arithmetic mean operators.

\section{Single-Container Maintenance Model by Fuzzy Geometric Programming Approach}

In the fuzzy container problem (2.3) fuzzy linear membership functiions for fuzzy objective and constraint goals are taken. The linear membership function for expected cost constraint is

$$
\mu_{J c}(J c(T))=\left\{\begin{array}{cl}
1 & \text { if } J c(T) \leq J c \\
\frac{J c+P_{J c}-J c(T)}{P_{J c}} & \text { if } J c \leq J c(T) \leq J c+P_{J c} \\
0 & \text { if } J c(T) \geq J c+P_{J c}
\end{array}\right.
$$

Its rough sketch is given in figure 2.

$<$ Figure $2>$

And the linear membership function for $M(T)$ is

$$
\mu_{M}(M(T))=\left\{\begin{array}{cl}
1 & \text { if } M(T) \leq L \\
\frac{L+P_{L}-M(T)}{P_{L}} & \text { if } L \leq M(T) \leq L+P_{L} \\
0 & \text { if } M(T) \geq L+P_{L}
\end{array}\right.
$$

Its rough sketch is given in figure 3.

$<$ Figure $3>$

Here fuzzy container problem(2.3) is described through different operators.

(i) Based on Max-Min Operator:

Through max-min operator, fuzzy model (2.3) reduces to

$\operatorname{Max}\left(1+\frac{L}{P_{L}}-\frac{T}{P_{L}}\right)$

subject to $\left(\frac{C_{P}}{T}+\left(\frac{C_{r}}{\beta^{\alpha}}\right) T^{\alpha-1}-J c\right) P_{L} \leq(T-L) P_{J_{C}}$

$$
T>0
$$

The above maximization problem can be obtained by solving following minimization problem

$\operatorname{Min} \sigma_{0} \frac{T}{P_{L}}$

subject to 
$\sigma_{1} C_{m 1} T^{-1}+\sigma_{2} C_{m 2} T^{\alpha-1}+\sigma_{3} C_{m 3} T \leq 1$

$T>0$

Where $C_{m 1}=\frac{P_{L} C_{P}}{J c P_{L}-L P_{J c}}, C_{m 2}=\frac{P_{L} C_{r}}{\left(J c P_{L}-L P_{J c}\right) \beta^{\alpha}}, C_{m 3}=\frac{P_{J c}}{J c P_{L}-L P_{J c}}$

It is a constraint signomial GP with $D D=4-1-1=2$

The Dual Problem (DP) of above Primal Geometric Problem (PGP) is as follows

$\operatorname{Max} d w_{m}(w)=\zeta_{0}\left[\left(\frac{1}{P_{L} w_{01}}\right)^{\sigma_{0} w_{01}}\left(\frac{C_{m 1} \lambda}{w_{11}}\right)^{\sigma_{1} w_{11}}\left(\frac{C_{m 2} \lambda}{w_{12}}\right)^{\sigma_{2} w_{12}}\left(\frac{C_{m 3} \lambda}{w_{13}}\right)^{\sigma_{3} w_{13}}\right]^{\zeta_{0}}$

subject to

$\sigma_{0} w_{01}=\zeta_{0}$

$\sigma_{0} w_{01}-\sigma_{1} w_{11}+\sigma_{2}(\alpha-1) w_{12}+\sigma_{3} w_{13}=0$

where $\lambda=\sigma_{1} w_{11}+\sigma_{2} w_{12}+\sigma_{3} w_{13}$

where $w_{01}, w_{1 i} \geq 0$ for $i=1,2,3$, Here $\zeta_{0}$ is assume $+1, \sigma_{0}=\sigma_{1}=\sigma_{2}=1$ and $\sigma_{3}=-1$

solving system of linear equations (5.2) in terms of $w_{12}$ and $w_{13}$ and putting in objective function (5.1) we get

$\operatorname{Max} d w_{m}\left(w_{12}, w_{13}\right)=\frac{1}{P_{L}}\left(\frac{C_{m 1}\left(1+\alpha w_{12}\right)}{1+(\alpha-1) w_{12}+w_{13}}\right)^{\left(1+(\alpha-1) w_{12}+w_{13}\right)}\left(\frac{C_{m 2}\left(1+\alpha w_{12}\right)}{w_{12}}\right)^{w_{12}}$

$\left(\frac{C_{m 3}\left(1+\alpha w_{12}\right)}{w_{13}}\right)^{-w_{13}}$

subject to $w_{12}, w_{13}>0$

To find the optimal solution of the above objective function, taking logarithm and then differentiating with respect to $w_{12}$ and $w_{13}$ and equating to zero we get

$$
\begin{gathered}
(\alpha-1) \log \left(C_{m 1}\left(1+\alpha w_{12}\right)\right)+\log \left(C_{m 2}\left(1+\alpha w_{12}\right)\right)+\frac{\alpha(\alpha-1)\left(w_{12}-w_{13}\right)}{1+\alpha w_{12}} \\
=(\alpha-1) \log \left(1+(\alpha-1) w_{12}+w_{13}\right)-\log w_{12}
\end{gathered}
$$

and

$\log \left(C_{m 1}\left(1+\alpha w_{12}\right)\right)+\log w_{13}=\log \left(1+(\alpha-1) w_{12}+w_{13}\right)+\log w_{12}+\log \left(C_{m 3}\left(1+\alpha w_{12}\right)\right)$

Solving the above system of non-linear equations (5.3) and (5.4), optimal dual values $w_{12}^{*}$ and $w_{13}^{*}$ are obtained and hence optimal dual value of $w_{11}^{*}$ is solved and so optimal dual value of the object function is $d w_{m}^{*}\left(w^{*}\right)$.

Now from primal dual relation $T^{*}=P_{L} d w^{*}\left(w^{*}\right)$ the optimal value of the primal variable can be obtained. By the help of the above optimal decision variable $T^{*}$, we obtain the optimal value of the primal objective function.

ii) Based on Arithmetic Mean Operator:

Through max-arithmetic mean operator, fuzzy model (2.3) reduces to

$\operatorname{Max}\left(1+\frac{L}{P_{L}}-\frac{T}{P_{L}}-\frac{C_{P}}{T P_{J c}}-\frac{1}{P_{j c}}\left(\frac{C_{r}}{\beta^{\alpha}}\right) T^{\alpha-1}+\frac{J c}{P_{j c}}+1\right) / 2$

The above maximization problem can be obtained by solving following minimization problem

$\operatorname{Min}\left(C_{a 1} T^{-1}+C_{a 2} T^{\alpha-1}+C_{a 3} T\right)$

$$
T>0
$$

Where $C_{a 1}=\frac{C_{P}}{P_{J c}}, C_{a 2}=\frac{C_{r}}{P_{J c} \beta^{\alpha}}, C_{3}=\frac{1}{P_{L}}$

It is an unconstraint posynomial GP with $D D=3-1-1=1$

The DP of above PGP is as follows

$\operatorname{Max} d w_{a}(w)=\left(\frac{C_{a 1}}{w_{01}}\right)^{w_{01}}\left(\frac{C_{a 2}}{w_{02}}\right)^{w_{02}}\left(\frac{C_{a 3}}{w_{03}}\right)^{w_{03}}$

$w_{01}+w_{02}+w_{03}=1$

$-w_{01}+(\alpha-1) w_{02}+w_{03}=0$

where $w_{01} \geq 0$ for $i=1,2,3$ 
Solving system of linear equations (5.6) in term of $w_{02}$ and putting in objective function (5.5) we get

$\operatorname{Max} d w_{a}\left(w_{02}\right)=\left(\frac{2 C_{a 1}}{1+(\alpha-2) w_{02}}\right)^{0.5\left(1+(\alpha-2) w_{02}\right)}\left(\frac{C_{a 2}}{w_{02}}\right)^{w_{02}}\left(\frac{2 C_{a 3}}{1-\alpha w_{02}}\right)^{0.5\left(1-\alpha w_{02}\right)}$

subject to $w_{02}>0$

To find the optimal solution of the above objective function, taking logarithm of the objective function and then differentiating with respect to $w_{02}$ and equating to zero we get

$(\alpha-2)\left\{\log 2 C_{a 1}-\log \left(1+(\alpha-2) w_{02}\right)\right\}+2 \log C_{a 2}=2 \log w_{02}+\alpha\left\{\log 2 C_{a 3}-\log \left(1-\alpha w_{02}\right)\right\}$

Solving the above non linear equations (5.7) optimal dual value of $w_{02}^{*}$ is obtained and hence optimal dual values of $w_{01}^{*}=0.5\left(1+(\alpha-2) w_{02}^{*}\right)$ and $w_{03}^{*}=0.5\left(1-\alpha w_{02}\right)$ are attained so, optimal value of the dual objective function is $d w_{a}^{*}\left(w^{*}\right)$

Now from primal dual relation $T^{*}=\frac{w_{01}^{*} d w^{*}\left(w^{*}\right)}{C_{a 1}}$, the optimal value of the primal variable can be obtained. By the help of the above optimal decision variable $T^{*}$, the optimal value of the primal objective function is achieved.

iii) Based on Geometric Mean Operator:

Through max-geometric mean operator, fuzzy model (2.3) reduces to

$\operatorname{Max}\left(\left(\frac{J c+P_{j c}}{P_{j c}}-\frac{C_{P}}{T P_{J c}}-\frac{1}{P_{j c}}\left(\frac{C_{r}}{\beta^{\alpha}}\right) T^{\alpha-1}\right) \times\left(\frac{L+P_{L}+T}{P_{L}}\right)\right)^{\frac{1}{2}}$

the above maximization problem can be obtained by solving following GP problem

$\operatorname{Min}\left\{\sigma_{01} C_{p 1} T^{-1}+\sigma_{02} C_{p 2} T^{\alpha-1}+\sigma_{03} C_{p 3} T+\sigma_{04} C_{p 4} T^{\alpha}\right\}$

$$
T>0
$$

Where $C_{p 1}=\frac{\left(L+P_{L}\right) C_{p}}{P_{L} P_{J c}}, C_{p 2}=\frac{\left(L+P_{L}\right) C_{r}}{P_{L} P_{J c}}, C_{p 3}=\frac{J c+P_{J c}}{P_{L} P_{J c}}, C_{p 4}=\frac{C_{r}}{P_{L} P_{J c} \beta^{\alpha}}$

It is an unconstraint signomial GP with $D D=4-1-1=2$

The dual problem of above primal problem is as follows

$\operatorname{Max} d w_{p}(w)=\zeta_{0}\left[\left(\frac{C_{p 1}}{w_{01}}\right)^{\sigma_{01} w_{01}}\left(\frac{C_{p 2}}{w_{02}}\right)^{\sigma_{02} w_{02}}\left(\frac{C_{p 3}}{w_{03}}\right)^{\sigma_{03} w_{03}}\left(\frac{C_{p 4}}{w_{04}}\right)^{\sigma_{04} w_{04}}\right]^{\zeta_{0}}$

subject to

$\sigma_{01} w_{01}+\sigma_{02} w_{02}+\sigma_{03} w_{03}+\sigma_{04} w_{04}=\zeta_{0}$

$-\sigma_{01} w_{01}+\sigma_{02}(\alpha-1) w_{02}+\sigma_{03} w_{03}+\sigma_{04} \alpha w_{04}=0$

where $w_{01} \geq 0$ for $i=1,2,3,4$, Here $\zeta_{0}$ is assume $+1, \sigma_{01}=\sigma_{02}=\sigma_{03}=1$ and $\sigma_{04}=-1$

Solving system of linear equations (5.9) in terms of $w_{02}$ and $w_{04}$ and putting in objective function (5.8) we get

$$
\begin{aligned}
& \operatorname{Max} d w_{p}(w)=\left(\frac{2 C_{p 1}}{1+(\alpha-2) w_{02}+(\alpha-1) w_{04}}\right)^{0.5\left(1+(\alpha-2) w_{02}+(\alpha-1) w_{04}\right)}\left(\frac{C_{p 2}}{w_{02}}\right)^{w_{02}} \times \\
&\left(\frac{2 C_{p 3}}{1-\alpha w_{02}-(1+\alpha) w_{04}}\right)^{0.5\left(1-\alpha w_{02}-(1+\alpha) w_{04}\right)}\left(\frac{C_{p 4}}{w_{04}}\right)^{w_{04}}
\end{aligned}
$$

To find the optimal solution of the above objective function, taking logarithm and then differentiating with respect to $w_{02}$ and $w_{04}$ and equating to zero, equations are as follows

$(\alpha-1) \log 2 C_{p 1}+2 \log C_{p 2}+\alpha \log \left(1+\alpha w_{02}-(\alpha+1) w_{04}\right.$

$=(\alpha-2) \log \left(1+(\alpha-2) w_{02}+(\alpha-1) w_{04}\right)+2 \log w_{02}+\alpha \log 2 C_{p 3}$

and

$4+(\alpha-1) \log 2 C_{p 1}+2 \log w_{04}+(\alpha+1) \log \left(1-\alpha w_{02}-(\alpha+1) w_{04}\right)$

$=(\alpha-1) \log \left(1+(\alpha-2) w_{04}+(\alpha-1) w_{04}\right)+2 \log C_{p 4}+(1-\alpha) \log 2 C_{p 3}$

Solving the above system of non-linear equations (5.10) and (5.11), optimal values $w_{02}^{*}$ and $w_{04}^{*}$ are obtained and hence other optimal value of dual variables are

$w_{01}^{*}=0.5\left(1+(\alpha-2) w_{02}^{*}+(\alpha-1) w_{04}^{*}\right)$ and $w_{02}^{*}=0.5\left(1-\alpha w_{02}^{*}-(1+\alpha) w_{04}^{*}\right)$ so optimal value of the dual objective function is $d w_{p}^{*}\left(w^{*}\right)$.

Now from primal dual relation $T^{*}=d w^{*}\left(w^{*}\right) P_{L}$, we obtain the optimal value of the primal variable. By the help of the above optimal decision variable $T^{*}$, the optimal value of the primal objective function is obtained. 


\section{Multi-container Maintenance Model by Fuzzy Geometric Programming Approach}

In the fuzzy container problem (2.4) and DD is higher so for problem MGP is applied through max-arithmetic mean operator with fuzzy linear membership functions for fuzzy objectives and constraint goals. The problem (2.4) is equivalent to the following fuzzy objective goal problem:

Find $T=\left(T_{1}, T_{2}, T_{3}, \cdots, T_{n}\right)$

$$
J c_{i}\left(T_{i}\right) \leq J c_{i} \quad \text { for } i=1,2,3, \cdots, n
$$

with the fuzzy constraint $M\left(T_{1}, T_{2}, \cdots, T_{n}\right) \underset{\sim}{\leq} L^{\prime}$

where $T_{i}>0 \quad$ for $i=1,2,3, \cdots, n$

In the above formulation, it is assumed that the average maintenance cost has a target $J c_{i}$ for the first item. such a situation may occur that it is compelled to enhance some more expenditure for maintenance say $P_{J_{c_{1}}}$ for the first item. similar cases may also happen for other cases. It is assumed that the objective goals are imprecise having a minimum targets $J c_{1}, J c_{2}, \cdots, J c_{n}$ with positive tolerances $P_{J c_{i}}, P_{J c_{2}}, \cdots, P_{J c_{n}}$ and the constraint goal having target $L^{\prime}$ with positive tolerance $P_{L^{\prime}}$. The above stated multi-item, multi-objective fuzzy maintenance model is solved by GP method.

The linear membership functions for the n-objective are

$$
\mu_{i}\left(J c_{i}\left(T_{i}\right)\right)=\left\{\begin{array}{cl}
1 & \text { if } J c_{i}\left(T_{i}\right) \leq J c_{i} \\
\frac{J c_{i}+P_{J c_{i}}-J c_{i}\left(T_{i}\right)}{P_{J c_{i}}} & \text { if } J c_{i} \leq J c_{i}\left(T_{i}\right) \leq J c_{i}+P_{J c_{i}} \\
0 & \text { if } J c_{i}\left(T_{i}\right) \geq J c_{i}+P_{J c_{i}}
\end{array}\right.
$$

And the linear membership function for $M\left(T_{1}, T_{2}, \cdots, T_{n}\right)$ is

$$
\mu\left(M\left(T_{1}, T_{2}, \cdots, T_{n}\right)\right)=\left\{\begin{array}{cl}
1 & \text { if } M\left(T_{1}, T_{2}, \cdots, T_{n}\right) \leq L^{\prime} \\
\frac{\left(L^{\prime}+P_{L^{\prime}}\right)-M\left(T_{1}, T_{2}, \cdots, T_{n}\right)}{P_{L^{\prime}}} & \text { if } L^{\prime} \leq M\left(T_{1}, T_{2}, \cdots, T_{n}\right) \leq L^{\prime}+P_{L^{\prime}} \\
0 & \text { if } M\left(T_{1}, T_{2}, \cdots, T_{n}\right) \geq L^{\prime}+P_{L^{\prime}}
\end{array}\right.
$$

MM and GM operators are not applied to avoid higher DD. Here AM operator for the problem (6.1) has been applied and thereby it is considered as

$\operatorname{Max} Y\left(T_{i}\right)=\left(\sum_{i=1}^{n} \mu_{i}\left(J c_{i}\left(T_{i}\right)\right)+\mu\left(M\left(T_{1}, T_{2}, \cdots, T_{n}\right)\right)\right) /(n+1)$

subject to

$\mu_{i}\left(J c_{i}\left(T_{i}\right)\right)=\frac{J c_{i}+P_{J c_{i}}-J c_{i}\left(T_{i}\right)}{P_{J c_{i}}} \quad$ for $i=1,2,3, \cdots, n$

$\mu\left(M\left(T_{1}, T_{2}, \cdots, T_{n}\right)\right)=\frac{L^{\prime}+P_{L^{\prime}}-M\left(T_{1}, T_{2}, \cdots, T_{n}\right)}{P_{L^{\prime}}}$

$\mu_{i}\left(J c_{i}\left(T_{i}\right)\right) \in[0,1] i=1,2,3, \cdots, n, \mu\left(M\left(T_{1}, T_{2}, \cdots, T_{n}\right)\right) \in[0,1]$ and $T_{i}>0$

It is sufficient to derive the minimization problem as

$\operatorname{Min} Z\left(T_{i}\right)=\sum_{i=1}^{n}\left(\frac{C_{p i}}{P_{J c i} T_{i}}+\frac{C_{r i}}{\beta^{\alpha} P_{j c i}} T_{i}^{\alpha-1}\right)+\frac{1}{P_{L^{\prime}}} \sum_{i=1}^{n} T_{i}=\sum_{i=1}^{n} X_{i}\left(T_{i}\right)$

such that $T_{i}>0$

for $i=1,2,3, \cdots, n$

where $X_{i}\left(T_{i}\right)=\left(\theta_{1 i} T_{i}^{-1}+\theta_{2 i} T_{i}^{\alpha-1}+\theta_{3 i} T_{i}\right)$ and $\theta_{1 i}=\frac{C_{p i}}{P_{J c i}}, \theta_{2 i}=\frac{C_{r i}}{\beta^{\alpha} P_{j c i}}$, and $\theta_{3 i}=\frac{1}{P_{L^{\prime}}}$

Here (6.3) is an unconstrained GP problem with $D D=(2 n-1)$, which is difficult to solve by formulating its dual problem for higher values of $n$. To reduce the DD, MGP method consider only the terms of $i^{\text {th }}$ function $(i=1,2,3, \cdots, n)$ instead of all the terms of the objective function. Here the number of terms in the $i^{\text {th }}$ function is there and the number of decision variables of the function is one. so DD is now reduced to one. According to this formulation, the DP is 
$\operatorname{Max} d w\left(w_{1}, w_{2}, w_{3}\right)=\prod_{i=1}^{n}\left[\left(\frac{\theta_{1 i}}{w_{1 i}}\right)^{w_{1 i}}\left(\frac{\theta_{2 i}}{w_{2 i}}\right)^{w_{2 i}}\left(\frac{\theta_{3 i}}{w_{3 i}}\right)^{w_{3 i}}\right]$

subject to normally and orthogonality conditions

$$
\begin{aligned}
& w_{1 i}+w_{2 i}+w_{3 i}=1 \\
& -w_{1 i}+(\alpha-1) w_{2 i}+w_{3 i}=0 \quad \text { for } i=1,2,3, \cdots, n
\end{aligned}
$$

where $w_{1 i}, w_{2 i}, w_{3 i} \geq 0$ for $i=1,2,3, \cdots, n$

solving system of linear equations (6.5)in term of $w_{2 i}$ and putting in objective function (6.4) and it becomes

$\operatorname{Max} d w\left(w_{2 i}\right)=\left(\frac{2 \theta_{1 i}}{1+(\alpha-2) w_{2 i}}\right)^{0.5\left(1+(\alpha-2) w_{2 i}\right)}\left(\frac{\theta_{2 i}}{w_{2 i}}\right)^{w_{2 i}}\left(\frac{2 \theta_{3 i}}{1-\alpha w_{2 i}}\right)^{0.5\left(1-\alpha w_{2 i}\right)}$

subject to $w_{2 i}>0$

Differentiate the objective function of (6.6) with respect to $w_{2 i}(i=1,2,3, \cdots, n)$ and the equating to zero we get

$(\alpha-2)\left\{\log 2 \theta_{1 i}-\log \left(1+(\alpha-2) w_{2 i}\right)\right\}+2 \log \theta_{2 i}=2 \log w_{2 i}+\alpha\left\{\log 2 \theta_{3 i}-\log \left(1-\alpha w_{2 i}\right)\right\}$

Solving the above non linear equation (6.7), we get optimal dual value of $w_{2 i}^{*}$. Hence the other dual values are $w_{1 i}^{*}=$ $0.5\left(1+(\alpha-2) w_{2 i}^{*}\right), w_{3 i}^{*}=0.5\left(1-\alpha w_{2 i}^{*}\right)$ and so optimal value of the dual objective function is $d w^{*}$

Now from primal dual relation, we can obtain the optimal value of the primal variable and primal objective function

$$
\begin{aligned}
& \begin{array}{cccc}
Z^{*} & = & n\left(d w^{*}\right)^{\frac{1}{n}} & \\
\frac{\theta_{1 i}}{T_{i}^{*} w_{1 i}^{*}} & = & \frac{Z^{*}}{n} & \text { for } i=1,2,3, \cdots, n
\end{array} \\
& \text { or } \frac{\theta_{2 i} T_{i}^{*}{ }^{\alpha-1}}{w_{2 i}^{*}}=\frac{Z^{*}}{n} \quad \text { for } i=1,2,3, \cdots, n
\end{aligned}
$$

By the help of the above optimal decision variable $T_{i}^{*}$, the optimal value of the primal objective functions is obtained as $J c_{i}^{*}\left(T_{i}^{*}\right)$

\section{Numerical Example}

\subsection{Numerical Exposure of Single Container maintenance problem}

Here the single container problem is considered for the numerical exposure as follows.

The coefficients and indices are taken as:

$C_{p}=100, C_{r}=10, \alpha=2, \beta=1, J c=65, P_{j c}=5, L=2.5, P_{L}=0.5, m=2.5$

The optimal solutions of the fuzzy model through FGP with three operators are presented in table 1.

From the above table the optimal objective value is better through max-arithmetic mean operator than the other two operators. Decision maker can take the decision when it is required to replace the container.

\subsection{Numerical Exposure of Multi-Container maintenance problem}

Considering there is three containers in the system of different corrective and preventive maintenance cost of each container. The input data are shown in the table 2.

Table 3 shown the optimal values $T_{i}^{*}$ with corresponding optimal value $J c_{i}^{*}\left(T_{i}^{*}\right)$ for $i=1,2,3$ solved by MGP through max-arithmetic mean operator of FGP. From the above table it is easy to say that MGP gives better result than the NLP.

\section{Conclusion}

In the earliest stage of system designing, the maintenance policy is usually made on precise data but in real life problem, available data is incomplete and inexact in nature. In this paper, maintenance problem of container model has been taken in view of maintenance work up to a certain level in fuzzy environment. This fuzzy maintainability of container problem is discussed through fuzzy geometric programming. Here fuzzy GP is illustrated through fuzzy decision-making processes. Three different operators namely, max-min, max-arithmetic mean, max-geometric mean are considered for this fuzzy GP. If situation demands it is easy to solve by FGP method for the single container and MGP methods for multi-container maintenance model considering different failure rates and shape parameters for different containers made of different materials.

There are more several operators based on different t-norms, this FGP may be developed using this operators.

Several reliability, maintenance and replacement models in fuzzy environment can be solved easily through fuzzy geometric programming than other fuzzy non-linear programming methods. 


\section{Appendix-I}

In the block policy, the time instant at which preventive maintenance occurs is renewal points. Cycle length is a determining quantity and the cycle cost is the sum of a preventive replacement $(\mathrm{Cp})$ at the end of the cycle and corrective repairs over the cycle length. Since failures are rectified through minimal repairs, the failures (and repairs) over a cycle occur according to a non-homogeneous Poisson process with an intensity function given by the failure rate of the item. As a result, the expected cycle cost (ECC) [3] is given by

$$
E C C=C_{p}+C_{r} \int_{0}^{T} r(x) d x
$$

Where $r(x)$ is the failure rate associated with the failure distribution $F(x)$, from the renewal reward theorem, it is obtained that $J c(T)=\frac{E C C}{E C L}=\frac{C_{p}+C_{r} \int_{0}^{T} r(x) d x}{T}$ where $E C L$ is the expected cycle length.

Harter and Moore (1976) give a comprehensive list of references dealing with the applicability of the Weibull distribution to model failure times for many different technical objects. Keceioglu (1991) explains the use of this distribution in modeling failure times for electron tubes, capacitors, ball bearings, leakage from batteries and many other applications. Now failure distribution, $F(x)$ is a Weibull distribution with shape parameter $\alpha$ and scale parameter $\beta$, i.e. failure rate is $r(x)=\frac{\alpha x^{\alpha-1}}{\beta^{\alpha}}$, then

$$
\begin{aligned}
J_{c}(T) & = & \frac{C_{p}}{T}+\frac{C_{r}}{T} \int_{0}^{T} \frac{\alpha x^{\alpha-1}}{\beta^{\alpha}} d x \\
& = & \frac{C_{p}}{T}+\frac{C_{r}}{\beta^{\alpha}} T^{\alpha-1}
\end{aligned}
$$

\section{References}

Abou-et-ata, M.O., \& Kotb, K.A.M. (1997). Multi-item EOQ inventory model with varying holding cost under two restrictions: a geometric programming approach. Production Planning Control, 8, 608-611.

Bellmann, R.E., \& Zadeh, L.A. (1970). Decision making in a fuzzy environment. Management Science, 17(4), B141B164.

Blischke, W.R., \& Murthy, D.N.P. (2000). Reliability Modeling, Prediction, and Optimization. New York, John Wiley \& Sons, Inc.

Cao B.Y. (1987). Solution and theory of question for a kind of fuzzy positive geometric program. Proc. 2nd IFSA Congress, Tokyo, 1, 205-208.

Cao, B.Y. (1993). Fuzzy geometric programming (I). Fuzzy Sets and Systems, 53, 135-153.

Cao, B.Y. (2002). Fuzzy Geometric Programming. Kluwer Academic Publishers, Netherlands.

Duffin, R. J., Peterson, E. L., \& Zener, C. (1967). Geometric Programming-Theory and Application. New York: John Wiley.

Govil, K.K. (1992). Optimal maintainability allocation using geometric programming method. Microelectronics Reliability, 32(1-2), 265-266.

Harter, H.L. \& Moore, A.H. (1976). An evaluation of exponential and weiblull test plans. IEEE Transactions on Reliability, R-25, 100-104.

Islam, S., \& Roy, T.K. (2005). Modified Geometric programming problem and its applications. Journal of Applied Mathematics and Computing, 17 (1-2), 121-144.

Keceioglu, D. (1991). Reliability Engineering Handbook. 1, Prentice Hall, Englewood Ciffs, NJ.

Passy, U., \& Wilde, D.J. (1967). Generalized polynomial optimization. SIAM Journal for Applied Mathematics, 15, 1344-1356.

Sakawa, M. (1993). Fuzzy sets and interactive multi-objective optimization. Plenum press, New York.

Zadeh, L.A. (1965). Fuzzy sets. Information and Control, 8, 338-353.

Zener, C. (1971). Engineering Design by Geometric Programming. New York: Wiley- Interscience.

Table 1. Optimal solutions of fuzzy single container model(2.3) 


\begin{tabular}{|c|c|c|}
\hline Operator & $T^{*}($ Year $)$ & $J_{c}^{*}\left(T^{*}\right)(\$)$ \\
\hline Max-min & 2.2222 & 67.2222 \\
\hline Max-arithmetic mean & 2.2360 & 67.0820 \\
\hline Max-geometric mean & 2.2249 & 67.1943 \\
\hline
\end{tabular}

Table 2. Input data of fuzzy multi-objective multi-containers model (2.4)

\begin{tabular}{|c|c|c|c|c|c|c|c|}
\hline $\mathrm{i}$ & $\begin{array}{c}J c_{i} \\
\left(\text { Minimum values of } J c_{i}\right)(\$)\end{array}$ & $\begin{array}{c}P_{J c_{i}} \\
\text { (Maximum tolerance) }\end{array}$ & $\alpha$ & $\beta$ & $L^{\prime}$ & $P_{L^{\prime}}$ & $m_{i}$ \\
\hline 1 & 64.8074 & 4.40 & 2 & 1 & 10 & 2.5 & 0.7 \\
\hline 2 & 59.3295 & 4.55 & 2 & 1 & 10 & 2.5 & 0.7 \\
\hline 3 & 69.2820 & 2.92 & 2 & 1 & 10 & 2.5 & 0.7 \\
\hline
\end{tabular}

Table 3. Optimal solution of fuzzy multi-objective multi-containers model (2.4)

\begin{tabular}{|c|c|c|c|c|}
\hline $\mathrm{i}$ & $\begin{array}{c}T_{i}^{*}(\text { Year }) \\
\text { MGP-method }\end{array}$ & $\begin{array}{c}J c_{i}^{*}\left(T_{i}^{*}\right)(\$) \\
\text { MGP-method }\end{array}$ & $\begin{array}{c}T_{i}^{*}(\text { Year }) \\
\text { NLP-method }\end{array}$ & $\begin{array}{c}J c_{i}^{*}\left(T_{i}^{*}\right)(\$) \\
\text { NLP-method }\end{array}$ \\
\hline 1 & 2.666339 & 66.04323 & 2.325641 & 68.40525 \\
\hline 2 & 2.701221 & 62.33209 & 2.513352 & 63.87307 \\
\hline 3 & 3.826449 & 72.05128 & 2.161007 & 72.20681 \\
\hline Total & & 200.4266 & & 204.4851 \\
\hline
\end{tabular}

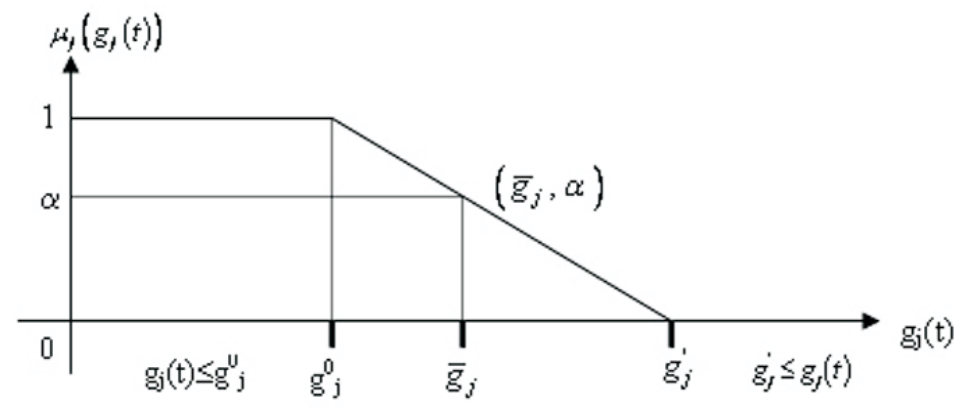

Figure 1. Linear membership function for $g_{j} \leq g_{j}^{0}$

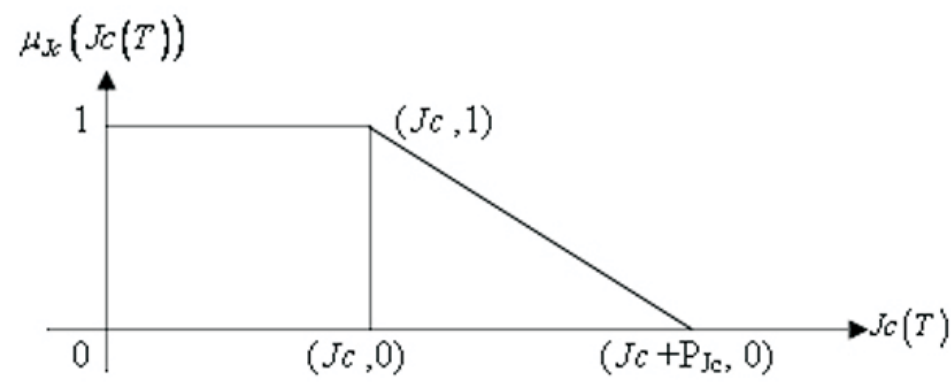

Figure 2. Linear membership function for $\tilde{\min } J_{c}(T)$ 


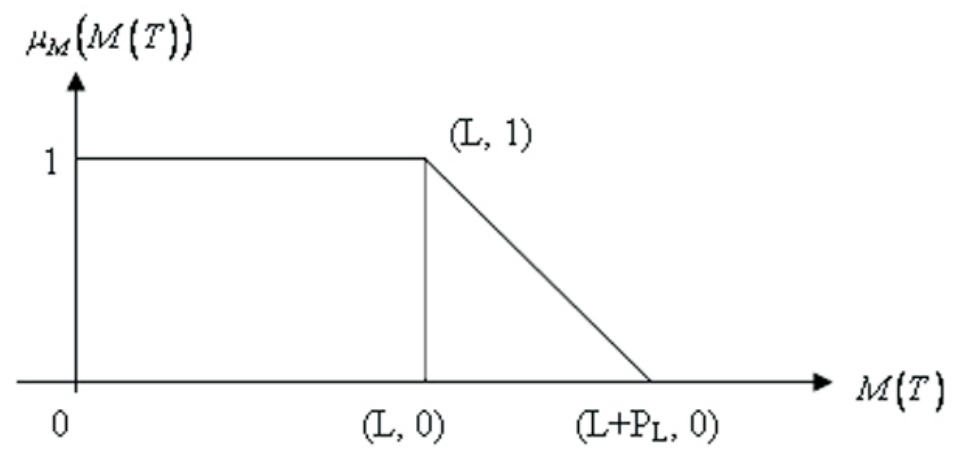

Figure 3. Membership function of $M(T) \leq L$ 